\title{
Neural network modeling for prediction of recurrence, progression, and hormonal non-remission in patients following resection of functional pituitary adenomas
}

\author{
Shane Shahrestani ${ }^{1,2}$ - Tyler Cardinal ${ }^{1}$ - Alexander Micko ${ }^{1,3}$ - Ben A. Strickland ${ }^{1}$ - Dhiraj J. Pangal ${ }^{1}$. \\ Guillaume Kugener ${ }^{1} \cdot$ Martin H. Weiss $^{1}$. John Carmichael ${ }^{1} \cdot$ Gabriel Zada $^{1}$
}

Accepted: 16 January 2021 / Published online: 2 February 2021

(c) The Author(s), under exclusive licence to Springer Science+Business Media, LLC part of Springer Nature 2021

\begin{abstract}
Purpose Functional pituitary adenomas (FPAs) cause severe neuro-endocrinopathies including Cushing's disease (CD) and acromegaly. While many are effectively cured following FPA resection, some encounter disease recurrence/progression or hormonal non-remission requiring adjuvant treatment. Identification of risk factors for suboptimal postoperative outcomes may guide initiation of adjuvant multimodal therapies.

Methods Patients undergoing endonasal transsphenoidal resection for $\mathrm{CD}$, acromegaly, and mammosomatotroph adenomas between 1992 and 2019 were identified. Good outcomes were defined as hormonal remission without imaging/biochemical evidence of disease recurrence/progression, while suboptimal outcomes were defined as hormonal non-remission or MRI evidence of recurrence/progression despite adjuvant treatment. Multivariate regression modeling and multilayered neural networks (NN) were implemented. The training sets randomly sampled $60 \%$ of all FPA patients, and validation/testing sets were $20 \%$ samples each.

Results 348 patients with mean age of 41.7 years were identified. Eighty-one patients (23.3\%) reported suboptimal outcomes. Variables predictive of suboptimal outcomes included: Requirement for additional surgery in patients who previously had surgery and continue to have functionally active tumor $(\mathrm{p}=0.0069 ; \mathrm{OR}=1.51,95 \% \mathrm{CI} 1.12-2.04)$, Preoperative visual deficit not improved after surgery $(\mathrm{p}=0.0033 ; \mathrm{OR}=1.12,95 \% \mathrm{CI} 1.04-1.20)$, Transient diabetes insipidus $(\mathrm{p}=0.013 ; \mathrm{OR}=1.27$, 95\%CI 1.05-1.52), Higher MIB-1/Ki-67 labeling index ( $\mathrm{p}=0.038 ; \mathrm{OR}=1.08,95 \% \mathrm{CI} 1.01-1.15)$, and preoperative low cortisol axis $(\mathrm{p}=0.040 ; \mathrm{OR}=2.72,95 \% \mathrm{CI} 1.06-7.01)$. The $\mathrm{NN}$ had overall accuracy of $87.1 \%$, sensitivity of $89.5 \%$, specificity of $76.9 \%$, positive predictive value of $94.4 \%$, and negative predictive value of $62.5 \%$. NNs for all FPAs were more robust than for CD or acromegaly/mammosomatotroph alone.

Conclusion We demonstrate capability of predicting suboptimal postoperative outcomes with high accuracy. NNs may aid in stratifying patients for risk of suboptimal outcomes, thereby guiding implementation of adjuvant treatment in high-risk patients.
\end{abstract}

Keywords Functional $\cdot$ Pituitary $\cdot$ Adenoma $\cdot$ Machine learning $\cdot$ Recurrence $\cdot$ Progression

Shane Shahrestani

shanesha@usc.edu

1 Department of Neurological Surgery, Keck School of Medicine, University of Southern California, Los Angeles, CA, USA

2 Department of Medical Engineering, California Institute of Technology, Pasadena, CA, USA

3 Department of Neurosurgery, Medical University of Vienna, Vienna, Austria

\section{Introduction}

Pituitary adenomas comprise approximately $15 \%$ of all primary central nervous system neoplasms [1,2]. According to a meta-analysis of Ezzat et al. PRL cell adenomas account for 30-60\%, followed by non-functioning adenomas (14-55\%), GH cell adenomas (8-15\%), ACTH cell adenomas $(2-6 \%)$ and TSH cell adenomas $(<1 \%)$ [3]. The majority of functional pituitary adenomas (FPAs) are prolactinomas, followed by somatotroph adenomas, corticotroph adenomas, thyrotroph adenomas, and rare 
functional gonadotroph adenomas [4]. While these tumors are almost always histologically benign, hormonal oversecretion is associated with significant morbidity and mortality in patients with FPAs [5-7]. Additionally, FPAs may exert mass effect on local neurovascular structures that can also significantly affect patient quality of life [7]. First-line therapy for non-prolactinoma FPAs is often transsphenoidal resection, which has been shown to be highly effective for tumor eradication and long-term hormonal remission, particularly in patients with Cushing's disease and acromegaly [5-9]. Specifically, endoscopic endonasal resection has been shown to be at least as effective as the traditional microscopic technique in leading to long-term remission with low complication rates [10-15].

While current literature extensively highlights the effectiveness and safety of endoscopic endonasal surgery, predictive factors for patient outcomes remain elusive, and would be useful to help guide postoperative adjuvant treatment including medical and radiation-based therapy. Studies have proposed the use of presenting patient symptoms including vision loss [16], tumor characteristics including size and invasion [9, 17-19], and pre- and postoperative endocrine lab values $[9,20]$ to predict long-term hormonal remission and tumor control. A recent systematic review of endoscopic pituitary surgery outcomes found older age to be predictive of postoperative complications, but suggested the need for more comprehensive data [21]. Another study created an algorithm for predicting recurrence and hormonal remission after endoscopic endonasal resection for treatment of Cushing's disease, yet suggested no single factor that could reliably predict outcomes [22]. The majority of current studies, however, lack consensus pertaining to predictors of outcomes in patients with FPAs. In this study, we examine outcomes in 348 cases of FPAs resected via microscopic and endoscopic endonasal transsphenoidal surgery (ETS) with long tern follow-up at a tertiary care center.

The aim of this study was to use multivariate analysis and NNM to determine reliable predictors of postoperative complications and patient outcomes to aid in physician and patient decision making.

\section{Methods}

\section{Patient sample}

This was a single center, Institutional Review Board (IRB HS-11-00,702) approved study. A retrospective chart review was conducted for all patients who underwent endonasal transsphenoidal resection of an FPA, including GH-secreting adenomas (acromegaly), ACTH-secreting adenomas (Cushing's disease), and mammosomatotroph (growth hormone + prolactin) adenomas. All patients with nonfunctioning or silent pituitary adenomas were excluded, as well as all patients with non-transsphenoidal tumor resection. Variables collected through chart review included patient demographics, presenting clinical symptoms, preoperative exam findings, history of prior treatment, tumor characteristics and immunostaining, tumor invasion, extent of resection, cerebrospinal fluid (CSF) leak status, perioperative complications, additional treatments, and follow-up outcomes including tumor recurrence, progression, or hormonal non-remission.

Endocrine remission was defined as in the latest consensus criteria: morning serum cortisol greater than $5 \mu \mathrm{g} / \mathrm{dl}$ alone or also with normal 24-h urinary free cortisol levels for ACTH-secreting adenomas, suppressed GH less than $0.4 \mathrm{ng} / \mathrm{ml}$ during oral glucose tolerance test or a random $\mathrm{GH}$ less than $1.0 \mathrm{ng} / \mathrm{ml}$ with normal insulin-like growth factor 1 (IGF-1), and normal prolactin and GH in mammosomatotroph adenomas [23-26].

"Good outcome" was defined as hormonal remission and no imaging or biochemical evidence of recurrence or progression following surgery, and subsequently were binarized and used as the primary outcome variable in this study. "Suboptimal outcome" was alternatively defined as cases with evidence of tumor recurrence, progression or hormonal non-remission despite multimodal therapy. A total of 348 patients with FPAs were identified between 1992 and 2019 matching inclusion and exclusion criteria and were included in the analysis.

\section{Data imputation}

Upon data inspection, several patients $(5.1 \%)$ had missing extent of resection data due to incomplete hospital records. As such, multiple imputation by chained equations (MICE) was implemented to fill in all missing values [27]. Post hoc data validation was performed to confirm that no missing values were included in the database at the time of analysis.

\section{Statistical analysis}

All statistical analysis was conducted in RStudio (Version 1.3.959).

\section{Multivariate analysis}

Multivariate regression modeling was performed to test whether patient-specific characteristics were correlated with good outcomes (hormonal remission without recurrence or progression). Covariates in the analysis included all patient demographic and perioperative variables to control for confounding factors. Using built-in functions, we exponentiated the coefficients of continuous variables analyzed within the multivariate models and interpreted them as odds-ratios. 
A two-sided $\mathrm{p}$ value $<0.05$ was considered statistically significant.

\section{Machine learning models}

Several multilayered neural network (NN) with 3 neurons in their hidden layer were implemented in RStudio using the 'neuralnet' package. NNs were used becausewe did not predict a simple linear relationship between our predictors and our outcomes, and NNs are able to perform well in nonlinear models. The training set for the $\mathrm{NN}$ including all patients was defined as a random $60 \%(n=208)$ sample of the total number of FPA patients, the validation set was a random sample of $20 \%(n=70)$, and the testing set was the remaining $20 \%(n=70)$ of these patients. Prediction with the validation set allowed for model tuning, while prediction with the test set represented the reported model output. Variables that showed significance on multivariate analysis were included as input variables into the $\mathrm{NN}$ model. Weights in our model were calculated using these back-propagation models. Bias terms were also obtained from our model. Smaller NNs generated for Cushing's disease and acromegaly + mammosomatotroph adenomas, with $60 \%$ of each patient pool being used as the training set and $40 \%$ as the testing set. The fully developed NN models were tested and confirmed using prediction and confusion matrices.

\section{Results}

\section{Patient characteristics}

The average patient age at the time of surgery was 41.7 $(\mathrm{SD} \pm 19.7)$ years and $63.8 \%$ of patients were female. The mean follow-up time was $68.2(\mathrm{SD} \pm 69.7$, Range: 1-290 months) months, with 307 (88.2\%) patients receiving the first operation for the tumor and $41(11.8 \%)$ receiving reoperation. FPA subtypes included 191 (54.9\%) GH-secreting adenomas, 146 (41.9\%) ACTH-secreting tumors and 11 (3.2\%) mammosomatotroph PAs. The average maximal tumor diameter was $6.5(\mathrm{SD} \pm 9.8)$ millimeters and 144/348 $(41.4 \%)$ of all tumors were macroadenomas $(>10 \mathrm{~mm})$. By far, the most common presenting clinical symptom was acromegaly $(\mathrm{n}=153,44.0 \%)$ followed by headache $(\mathrm{n}=77$, $22.1 \%)$ and vision loss $(\mathrm{n}=29,8.3 \%)$, and upon endocrine evaluation 13 (3.8\%) patients had a low gonadotrope axis, and $1(0.29 \%)$ patient had panhypopituitarism. The mean hospital length of stay (LOS) was $2.6(\mathrm{SD} \pm 3.2)$ days. On magnetic resonance imaging (MRI) evaluation, 87 (25.0\%) FPAs were found to invade into the cavernous sinus, 50 (14.4\%) FPAs extended into the suprasellar space, and 25 (7.2\%) had infrasellar invasion into the sphenoid sinus or clivus. Intraoperative extent of resection showed 287 (82.5\%) cases of gross-total resection (GTR), 43 (12.4\%) with subtotal resection (STR), and 18 (5.1\%) did not report intraoperative extent of resection in the patient record. These missing data were imputed using MICE, yielding a total of 305 (87.6\%) GTR and 43 (12.4\%) STR. Tumor progression developed in $6(1.7 \%)$ patients and tumor recurrence developed in 25 (7.2\%) patients. Hormonal nonremission was seen in 54 patients (15.5\%). Removing overlapping patients, a total of $81(23.3 \%)$ patients had suboptimal outcomes within the entire cohort (Table 1).

\section{Multivariate analysis findings}

Multivariate analysis for relevant patient characteristics revealed several factors that were associated with suboptimal postoperative outcomes in patients with FPAs. ORs above 1.00 suggested higher odds of a "suboptimal" outcome (i.e., lower odds of a good outcome) and ORs below 1.00 suggested lower odds of a "suboptimal" outcome (i.e., higher odds of a good outcome).Variables predictive of suboptimal outcomes included: Requirement for additional surgery in patients who previously had surgery and continue to have functionally active tumor $(\mathrm{p}=0.0069$; $\mathrm{OR}=1.51$, 95\%CI 1.12-2.04), Preoperative visual deficit not improved after surgery $(\mathrm{p}=0.0033$; $\mathrm{OR}=1.12,95 \% \mathrm{CI} 1.04-1.20)$,

Table 1 Patient demographics

\begin{tabular}{lc}
\hline & $\begin{array}{l}\text { FPA patient } \\
\text { demographics(n=348) }\end{array}$ \\
\hline Age (years) & $41.7(\mathrm{SD} \pm 19.7)$ \\
Sex & $222(63.8 \%)$ \\
Female, $\mathrm{n}(\%)$ & $126(36.2 \%)$ \\
Male, $\mathrm{n}(\%)$ & $68.2(\mathrm{SD} \pm 69.7)$ \\
Mean follow-up time (months) & $6.5(\mathrm{SD} \pm 9.8)$ \\
Maximal tumor diameter (mm) & \\
Pituitary adenoma subtype & $191(54.9 \%)$ \\
GH-secreting, $\mathrm{n}(\%)$ & $146(41.9 \%)$ \\
ACTH-secreting, $\mathrm{n}(\%)$ & $11(3.2 \%)$ \\
Mammosomatotroph, $\mathrm{n}(\%)$ & \\
Tumor invasion & $87(25.0 \%)$ \\
Cavernous sinus, $\mathrm{n}(\%)$ & $50(14.4 \%)$ \\
Suprasellar space, $\mathrm{n}(\%)$ & $25(7.2 \%)$ \\
Infrasellar space, $\mathrm{n}(\%)$ & \\
Extent of resection & $287(82.5 \%)$ \\
Gross-total, $\mathrm{n}(\%)$ & $43(12.4 \%)$ \\
Sub-total, $\mathrm{n}(\%)$ & \\
Suboptimal outcomes & $25(7.2 \%)$ \\
Recurrence, $\mathrm{n}(\%)$ & $6(1.7 \%)$ \\
Progression, $\mathrm{n}(\%)$ & $54(15.5 \%)$ \\
Hormonal non-remission & $81(23.3 \%)$ \\
Non-overlapping suboptimal outcomes & \\
\hline
\end{tabular}


Transient diabetes insipidus (DI) $(\mathrm{p}=0.013$; OR $=1.27$, 95\%CI 1.05-1.52), Higher MIB-1/Ki-67 labeling index percentage $(\mathrm{p}=0.038 ; \mathrm{OR}=1.08,95 \% \mathrm{CI} 1.01-1.15)$, and

Table 2 Multivariate analysis results for "suboptimal" postoperative outcomes

\begin{tabular}{llrr}
\hline & Odds ratio & $\begin{array}{l}\text { 95\% } \\
\text { Confidence } \\
\text { interval }\end{array}$ & P value \\
& & $1.12-2.04$ & 0.0069 \\
\hline $\begin{array}{l}\text { Additional treatment with } \\
\quad \text { Surgery }\end{array}$ & 1.51 & $1.10-1.26$ & $<0.0001$ \\
MRI at last clinical follow-up & 1.78 & $1.04-1.20$ & 0.0033 \\
Preoperative visual deficit not & 1.12 & $1.05-1.52$ & 0.013 \\
$\quad$ improved after surgery & & $0.96-0.99$ & 0.0081 \\
Transient diabetes Insipidus & 1.27 & $1.01-1.15$ & 0.038 \\
Hospital length of stay (days) & 0.97 & $1.06-7.01$ & 0.040 \\
MIB-1/Ki-67 labeling Index \% & 1.08 & $0.17-0.77$ & 0.0090 \\
Low cortisol axis & 2.72 & $0.052-0.53$ & 0.0028 \\
Low GH axis & 0.36 & $1.02-1.73$ & 0.038 \\
Panhypopituitarism & 0.17 & $0.30-0.96$ & 0.037 \\
Acromegaly & 1.33 & & \\
Prior craniotomy & 0.54 & & \\
\hline
\end{tabular}

preoperative low cortisol axis $(\mathrm{p}=0.040 ; \mathrm{OR}=2.72,95 \% \mathrm{CI}$ 1.06-7.01).

Conversely, variables predictive of good outcomes included: History of prior craniotomy $(\mathrm{p}=0.037$; $\mathrm{OR}=0.54$, 95\% CI 0.30-0.96), Longer hospital LOS $(\mathrm{p}=0.0081$; $\mathrm{OR}=0.97,95 \% \mathrm{CI} 0.96-0.99)$, preoperative low GH axis $(\mathrm{p}=0.0090 ; \mathrm{OR}=0.36,95 \% \mathrm{CI} 0.17-0.77)$, and preoperative panhypopituitarism $(\mathrm{p}=0.0028 ; \mathrm{OR}=0.17,95 \% \mathrm{CI}$ 0.052-0.53) (Table 2). ORs for continuous variables were interpreted as an increase in odds for each unit change in a variable (i.e., 1 day for LOS, $1 \%$ for MIB-1/Ki-67 labeling index \%, etc.). Tumor invasion, Knosp scoring, immunostaining, hospital type, CSF leak, medications, age, and sex were not found to be significant. All significant findings were used as inputs into our multilayered NN model.

\section{Neural networks}

The NN generated from significant variables from multivariate analysis using the training set of 208 PAs is shown in Fig. 1. We implemented two testing methods to confirm the accuracy of our NN model. First, we plotted the receiver operating characteristic (ROC) curve, with the

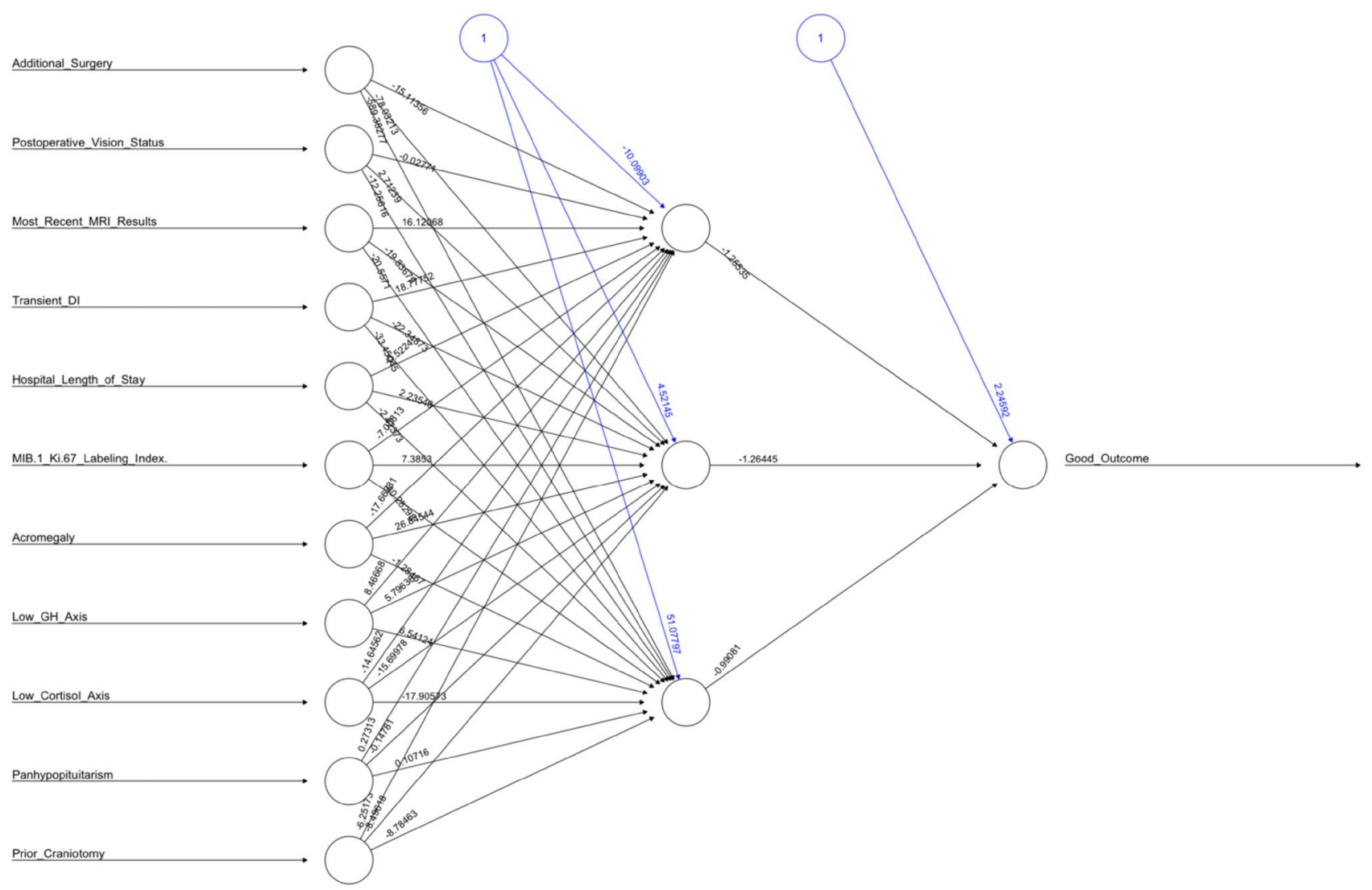

Fig. 1 Visualization of the most accurate computed neural network model with 3 hidden layer neurons constructed for all patients. Black lines represent weights as calculated by the back propagation algorithm, and blue lines represent calculated bias terms 
corresponding area under the curve (AUC) value (Fig. 2). With an AUC of 0.917, we see that the NN is able to correctly predict a large majority of patient outcomes with success. A regression line is also drawn to visually represent NN performance. Additionally, we present a confusion

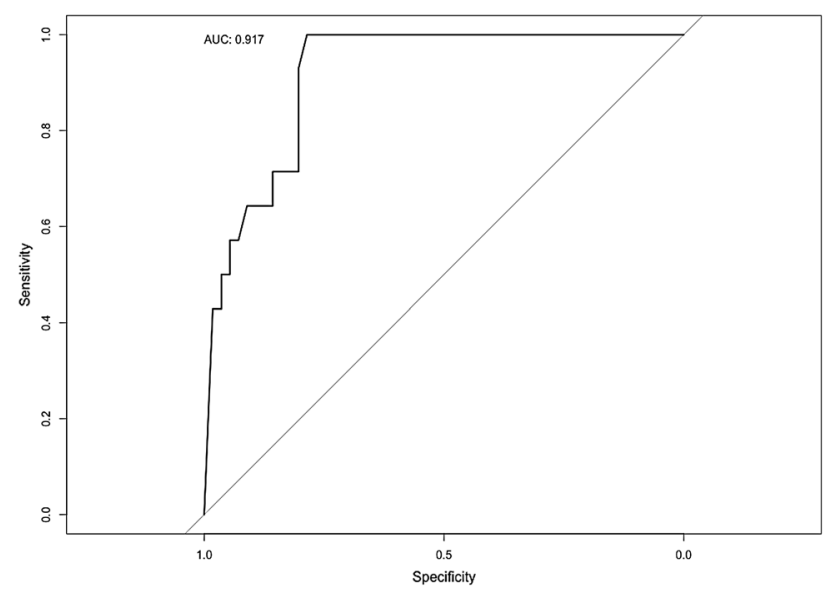

Fig. 2 Receiver operating characteristic (ROC) curve of the computed neural network for all patients. The area under the curve (AUC) is 0.917 matrix generated from applying the computed NN model to the training dataset. By doing so, we show that our NN model had an overall accuracy of $87.1 \%$, sensitivity of $89.5 \%$, specificity of $76.9 \%$, positive predictive value (PPV) of $94.4 \%$, and negative predictive value (NPV) of $62.5 \%$ (Table 3).

Two additional NNs were generated to evaluate whether predictive capabilities of our model would improve if the data included a homogenous sample of pituitary pathologies. Both the NNs generated for Cushing's disease (Table 3) and acromegaly + mammosomatotroph adenomas (Table 3 ) were outperformed by the aforementioned NN generated for the entire cohort. This finding suggests that heterogenous samples of FPA patients, with sufficient statistical power, may be used to accurately predict postoperative outcomes with high accuracy.

\section{Discussion}

We developed a multilayered neural network for predicting tumor recurrence, progression, or hormonal non-remission in a large cohort of patients whom underwent surgical resection of a FPA causing CD or acromegaly. Through

Table 3 Confusion matrices for neural network models

\begin{tabular}{|c|c|c|c|}
\hline \multicolumn{4}{|c|}{ Confusion matrix for neural network model—all patients } \\
\hline & Predicted "Good" outcome & Predicted "Suboptimal" outcome & \\
\hline $\begin{array}{l}\text { Actual "Good" } \\
\text { outcome }\end{array}$ & 51 & 6 & Sensitivity: $89.5 \%$ [81.3\%-97.7\%] \\
\hline \multirow{2}{*}{$\begin{array}{l}\text { Actual "Suboptimal" } \\
\text { outcome }\end{array}$} & 3 & 10 & Specificity: $76.9 \%$ [56.2\%-97.6\%] \\
\hline & $\begin{array}{l}\text { Positive predictive value: } 94.4 \% \\
{[88.4 \%-1.00 \%]}\end{array}$ & $\begin{array}{l}\text { Negative predictive value: } 62.5 \% \text { [ } 36.2 \%- \\
88.8 \% \text { ] }\end{array}$ & Accuracy: $87.1 \%$ [79.2\%-95.0\%] \\
\hline \multicolumn{4}{|c|}{ Confusion matrix for neural network model—cushing's disease } \\
\hline & Predicted "Good" outcome & Predicted "Suboptimal" outcome & \\
\hline $\begin{array}{l}\text { Actual "Good" } \\
\text { outcome }\end{array}$ & 45 & 3 & Sensitivity: $93.8 \%$ [87.1\%-99.9\%] \\
\hline \multirow{2}{*}{$\begin{array}{l}\text { Actual "Suboptimal" } \\
\text { outcome }\end{array}$} & 5 & 6 & Specificity: $45.5 \%[13.0 \%-78.0 \%]$ \\
\hline & $\begin{array}{l}\text { Positive predictive value: } 90.0 \% \\
\text { [81.5\%-98.5\%] }\end{array}$ & $\begin{array}{l}\text { Negative predictive value: } 66.7 \% \text { [38.8\%- } \\
94.6 \%]\end{array}$ & Accuracy: $86.4 \%$ [77.7\%-95.1\%] \\
\hline \multicolumn{4}{|c|}{ Confusion matrix for neural network model-acromegaly + mammosomatotroph } \\
\hline & Predicted "Good" outcome & Predicted "Suboptimal" outcome & \\
\hline $\begin{array}{l}\text { Actual "Good" } \\
\text { outcome }\end{array}$ & 48 & 15 & Sensitivity: $71.4 \%[59.2 \%-83.6 \%]$ \\
\hline \multirow{2}{*}{$\begin{array}{l}\text { Actual "Suboptimal" } \\
\text { outcome }\end{array}$} & 5 & 13 & Specificity: $72.2 \%$ [55.6\%-88.8\%] \\
\hline & $\begin{array}{l}\text { Positive predictive value: } 90.6 \% \\
\text { [83.4\%-97.8\%] }\end{array}$ & $\begin{array}{l}\text { Negative predictive value: } 46.4 \% \text { [23.4\%- } \\
69.4 \% \text { ] }\end{array}$ & Accuracy: $75.3 \%$ [65.9\%-84.7\%] \\
\hline
\end{tabular}

95\% confidence intervals shown in brackets 
multivariate analysis we narrow our search for statistically significant patient variables that predicted a good outcome, which we then fed into our NN for predictive testing. These variables included MRI imaging during last clinical followup reviewed by radiologist or neurosurgeon, DI, tumor characteristics, abnormal preoperative endocrine testing, prior craniotomy, and hospital type. By doing so, we developed a highly accurate NN prediction system, with acceptable sensitivity, specificity, PPV, and NPV. Findings from this study may help to guide future decision-making following surgery for FPAs as it pertains to adjuvant medical and radiationbased treatments.

Previous studies have shown that machine learning methods may be good predictors of poor postoperative outcomes following neurosurgery. Nadezhdina et al. have developed artificial neural networks (ANN) capable of predicting recurrence and remission in patients with Cushing disease after transnasal adenomectomy [22]. While their ANN is robust for the case of Cushing disease, it is not generalizable to all FPAs. Furthermore, several contemporary studies have proven the utility of machine learning models in predicting surgical extent of resection of pituitary adenomas [28, 29]. Similarly, Hollon et al. have demonstrated the use of supervised machine learning in predicting early outcomes in a mixed cohort of surgically treated functional and nonfunctional pituitary adenomas. After analysis, they report an accuracy of $87.0 \%$ which is nearly identical to the accuracy of the NN developed in this study [30].

Our findings on multivariate analysis suggest that longer hospital LOS, low preoperative GH axis, panhypopituitarism, and a history of a prior craniotomy may reduce the odds of a suboptimal outcome. Conversely, additional treatment with surgery, stable vision status following surgery, transient DI, increased MIB-1/Ki-67 labeling index percentage, low preoperative cortisol axis, and a preoperative diagnosis of acromegaly were shown to increase the odds of a suboptimal outcome. Transient DI is a known complication of pituitary surgery and has been shown to be proportional to the degree of damage to the pituitary gland [31, 32]. As a result, more aggressive tumors with a high likelihood of recurrence or progression may require increased margins of resection, thus increasing the rates of transient DI.

In the case of patients receiving surgical treatment for acromegaly, prior literature suggests a disappointing overall cure rate of $33 \%$ [33], with mortality being directly related with postoperative GH concentrations [34, 35]. Our results suggest similar findings, with the diagnosis of acromegaly increasing the odds of suboptimal outcomes and low preoperative $\mathrm{GH}$ axis reducing the odds of suboptimal outcomes. Additionally, the finding that shorter hospital stays may be predictive of suboptimal outcomes likely stems from the fact that patients with Cushing's disease are kept in the hospital longer than patients with acromegaly to monitor hormonal remission. Since our data suggests that acromegaly patients, who have shorter LOS compared to Cushing's patients, have worse overall outcomes, multivariate modelling likely associated reduced LOS with suboptimal outcomes. Furthermore, several studies have suggested that the MIB-1/Ki-67 labeling index percentage may be directly correlated with suboptimal postoperative outcomes in a variety of tumors, including pituitary tumors [36-39].

Lastly, we are hopeful that a robust NN capable of catching patients who may exhibit tumor recurrence, progression, or hormonal non-remission may allow for patient risk stratification. Specifically, patients at highest risk of suboptimal postsurgical outcomes may be more closely monitored and offered MRI imaging and hormonal testing more frequently during follow up visits. Through these measures, it may be possible to catch tumor recurrence, progression, and hormonal remission at an earlier stage to prevent significant and permanent complications in the patient.

\section{Limitations}

This study has the limitations associated with the retrospective character of this cohort study, in which our data is limited by the quality and depth of the records kept for patients included in this series. While retrospective chart review allowed for the collection of a great amount of data for each patient, we lack data on patient-specific risk factors such as nutritional status, drug use, and frailty [40]. Furthermore, patients developing suboptimal outcomes outside of our follow-up interval may not be included in this analysis, and as such, our analysis may underreport suboptimal outcomes. Lastly, data included in this study are from a single institution's county and academic hospital, and future studies with more patients that includes multi-center data and longer follow-up is warranted to confirm these findings.

\section{Conclusion}

Machine learning has proved to be an invaluable tool in medicine, allowing for detailed data analysis and disease-state prediction. By applying multilayered $\mathrm{NN}$ to data gathered from patients surgically treated for FPAs, we successfully created a robust prediction algorithm for adenoma recurrence, progression, and hormonal non-remission. As such, further investigation through larger case series should be explored to improve the accuracy and predictive capabilities of NNs in determining postoperative outcomes following resection of an FPA. 


\section{References}

1. Ostrom QT, Cioffi G, Gittleman H et al (2019) CBTRUS statistical report: primary brain and other central nervous system tumors diagnosed in the United States in 2012-2016. Neuro Oncol 21:v1-v100

2. Melmed S (2011) Pathogenesis of pituitary tumors. Nat Rev Endocrinol 7:257-266

3. Melmed S (2020) Pituitary-tumor endocrinopathies. N Engl J Med 382:937-950

4. Aflorei ED, Korbonits M (2014) Epidemiology and etiopathogenesis of pituitary adenomas. J Neurooncol 117:379-394

5. Akin S, Isikay I, Soylemezoglu F et al (2016) Reasons and results of endoscopic surgery for prolactinomas: 142 surgical cases. Acta Neurochir 158:933-942

6. Kim JH, Hur KY, Lee JH et al (2017) Outcome of endoscopic transsphenoidal surgery for acromegaly. World Neurosurg 104:272-278

7. Mehta GU, Lonser RR (2017) Management of hormone-secreting pituitary adenomas. Neuro Oncol 19:762-773

8. Campbell PG, Kenning E, Andrews DW et al (2010) Outcomes after a purely endoscopic transsphenoidal resection of growth hormone-secreting pituitary adenomas. Neurosurg Focus 29:E5

9. Han Y-L, Chen D-M, Zhang C et al (2018) Retrospective analysis of 52 patients with prolactinomas following endoscopic endonasal transsphenoidal surgery. Medicine 97:e13198

10. Berker M, Hazer DB, Yücel T et al (2012) Complications of endoscopic surgery of the pituitary adenomas: analysis of 570 patients and review of the literature. Pituitary 15:288-300

11. Broersen LHA, Biermasz NR, van Furth WR et al (2018) Endoscopic vs. microscopic transsphenoidal surgery for Cushing's disease: a systematic review and meta-analysis. Pituitary 21:524-534

12. Gondim JA, Schops M, de Almeida JPC et al (2010) Endoscopic endonasal transsphenoidal surgery: surgical results of 228 pituitary adenomas treated in a pituitary center. Pituitary 13:68-77

13. Paluzzi A, Fernandez-Miranda JC, Tonya Stefko S et al (2014) Endoscopic endonasal approach for pituitary adenomas: a series of 555 patients. Pituitary 17:307-319

14. Todeschini AB, Santos ARLD, Dolci RLL et al (2019) Long term follow-up after endoscopic endonasal approach for the treatment of cushing's disease. J Neurol Surg B Skull Base 80:306-309

15. Wang F, Zhou T, Wei S et al (2015) Endoscopic endonasal transsphenoidal surgery of 1166 pituitary adenomas. Surg Endosc 29:1270-1280

16. Nayak P, Montaser AS, Hu J et al (2018) Predictors of postoperative diabetes insipidus following endoscopic resection of pituitary adenomas. J Endocr Soc 2:1010-1019

17. Babu H, Ortega A, Nuno M et al (2017) Long-term endocrine outcomes following endoscopic endonasal transsphenoidal surgery for acromegaly and associated prognostic factors. Neurosurgery $81: 357-366$

18. Hofstetter CP, Nanaszko MJ, Mubita LL et al (2012) Volumetric classification of pituitary macroadenomas predicts outcome and morbidity following endoscopic endonasal transsphenoidal surgery. Pituitary 15:450-463

19. Zhang K, Shen M, Qiao N et al (2020) Surgical outcomes and multidisciplinary management strategy of Cushing's disease: a single-center experience in China. Neurosurg Focus 48:E7

20. Sarkar S, Rajaratnam S, Chacko G, et al (2016) Pure endoscopic transsphenoidal surgery for functional pituitary adenomas: outcomes with Cushing's disease. Acta Neurochir 158:77-86; discussion 86

21. Lobatto DJ, de Vries F, Zamanipoor Najafabadi AH et al (2018) Preoperative risk factors for postoperative complications in endoscopic pituitary surgery: a systematic review. Pituitary 21:84-97
22. Nadezhdina EY, Rebrova OY, Grigoriev AY et al (2019) Prediction of recurrence and remission within 3 years in patients with Cushing disease after successful transnasal adenomectomy. Pituitary 22:574-580

23. Micko ASG, Wöhrer A, Höftberger R et al (2017) MGMT and MSH6 immunoexpression for functioning pituitary macroadenomas. Pituitary 20:643-653

24. Biller BMK, Grossman AB, Stewart PM et al (2008) Treatment of adrenocorticotropin-dependent Cushing's syndrome: a consensus statement. J Clin Endocrinol Metab 93:2454-2462

25. Katznelson L, Laws ER Jr, Melmed S et al (2014) Acromegaly: an endocrine society clinical practice guideline. J Clin Endocrinol Metab 99:3933-3951

26. Melmed S, Casanueva FF, Hoffman AR et al (2011) Diagnosis and treatment of hyperprolactinemia: an Endocrine Society clinical practice guideline. J Clin Endocrinol Metab 96:273-288

27. van Buuren S, Groothuis-Oudshoorn K (2011) mice: Multivariate Imputation by Chained Equations inR. J Statist Software 45

28. Staartjes VE, Serra C, Muscas G et al (2018) Utility of deep neural networks in predicting gross-total resection after transsphenoidal surgery for pituitary adenoma: a pilot study. Neurosurg Focus 45:E12

29. Serra C, Staartjes VE, Maldaner N et al (2018) Predicting extent of resection in transsphenoidal surgery for pituitary adenoma. Acta Neurochir 160:2255-2262

30. Hollon TC, Parikh A, Pandian B et al (2018) A machine learning approach to predict early outcomes after pituitary adenoma surgery. Neurosurg Focus 45:E8

31. Woods C, Thompson CJ (2008) Risk of diabetes insipidus after pituitary surgery. Expert Rev Endocrinol Metab 3:23-27

32. Carman N, Kay C, Petersen A et al (2019) Transient central diabetes insipidus after discontinuation of vasopressin. Case Rep Endocrinol 2019:4189525

33. Gittoes NJL, Sheppard MC, Johnson AP, Stewart PM (1999) Outcome of surgery for acromegaly - the experience of a dedicated pituitary surgeon. QJM 92:741-745

34. Jenkins D, O'Brien I, Johnson A et al (1995) The Birmingham pituitary database: auditing the outcome of the treatment of acromegaly. Clin Endocrinol 43:517-522

35. Swearingen B (1998) Long-term mortality after transsphenoidal surgery and adjunctive therapy for acromegaly. J Clin Endocrinol Metab 83:3419-3426

36. Fusco A, Zatelli MC, Bianchi A et al (2008) Prognostic significance of the $\mathrm{Ki}-67$ labeling index in growth hormone-secreting pituitary adenomas. J Clin Endocrinol Metab 93:2746-2750

37. Widhalm G, Wolfsberger S, Preusser M et al (2009) Residual nonfunctioning pituitary adenomas: prognostic value of MIB-1 labeling index for tumor progression. J Neurosurg 111:563-571

38. Shimura T, Kofunato Y, Okada R et al (2016) MIB-1 labeling index, $\mathrm{Ki}-67$, is an indicator of invasive intraductal papillary mucinous neoplasm. Mol Clin Oncol 5:317-322

39. Byun S-S, Lee M, Hong SK, Lee H (2019) Elevated Ki-67 (MIB1) expression as an independent predictor for unfavorable pathologic outcomes and biochemical recurrence after radical prostatectomy in patients with localized prostate cancer: A propensity score matched study. PLoS ONE 14:e0224671

40. Shahrestani S, Ballatori AM, Chen XT et al (2020) Analysis of modifiable and nonmodifiable risk factors in patients undergoing pituitary surgery. J Neurosurg. https://doi. org/10.3171/2020.4.JNS20417

Publisher's Note Springer Nature remains neutral with regard to jurisdictional claims in published maps and institutional affiliations. 